\title{
苯乙烯的氧化三氟甲硫基化反应
}

\author{
王建勇 ${ }^{\dagger}, a, b$ 马 岗 ${ }^{\dagger}, a$ 李 彦 ${ }^{a}$ 王细胜 ${ }^{*}, a$ \\ ( ${ }^{a}$ 中国科学技术大学化学系 合肥微尺度物质科学国家研究中心 合肥 230026) \\ $\left({ }^{b}\right.$ 齐鲁工业大学(山东省科学院)轻工科学与工程学院 济南 250353)
}

\begin{abstract}
摘要 使用稳定易得的三氟甲硫基银作为 “三氟甲硫基” 源, 通过苯乙烯的三氟甲硫基化/氧化反应, 合成了一系列多 取代 $\alpha$-三氟甲硫基苯乙酮. 以二甲基亚砜和氯仿的为溶剂，过硫酸钾为氧化剂，通过自由基历程进行双官能化反应，在 温和条件下, 以中等到良好的收率实现了一系列不同取代的苯乙烯的氧化三氟甲硫基化的高效转化, 为 $\alpha$-三氟甲硫基 苯乙酮的合成提供了一种简单、步骤经济的有效合成方法.

关键词＼cjkstart苯乙烯; 三氟甲硫基化; 自由基
\end{abstract}

\section{Trifluoromethylthiolation/Oxidation of Styrenes for Facile Synthesis of $\alpha$-Trifluoromethylthio Acetophenons}

\author{
Wang, Jian-Yong ${ }^{a, b, \dagger} \quad$ Ma, Lan ${ }^{a, \dagger} \quad \operatorname{Li}$, Yan $^{a} \quad$ Wang, Xi-Sheng ${ }^{*, a}$ \\ ( ${ }^{a}$ Hefei National Laboratory for Physical Sciences at the Microscale \& Department of Chemistry, \\ University of Science and Technology of China, Hefei 230026) \\ ( ${ }^{b}$ School of Light Industry and Engineering, Qilu University of Technology (Shandong Academy of Sciences), Jinan 250353)
}

\begin{abstract}
Trifluoromethylthiolation/oxidation of styrenes with easiy-handling $\mathrm{AgSCF}_{3}$ used as the trifluoromethylthiolating reagent has been described, which furnished a series of $\alpha$-trifluoromethylthio acetophenons. A variety of substituted styrene could be bifunctionalized via radical process in a mixed solvent of dimethyl sulfoxide (DMSO) and $\mathrm{CHCl}_{3}$, with potassium persulfate used as the oxidant under mild conditions. A simple and efficent method for the facile construction of various $\alpha$-trifluoromethylthio acetophenons is thus demonstrated.
\end{abstract}

Keywords styrene; trifluoromethylthiolation; radical

经过多年的发展，含氟有机化合物被广泛应用于材 料、医药和农药等领域 ${ }^{[1]}$. 作为含氟有机化合物的重要 一员，三氟甲硫基化合物近来受到了科研人员广泛关 注, 这主要是由于三氟甲基硫基团具有强拉电性和亲脂 性, 可以显著改变非氟母体分子的生物穿透性、化学和 代谢稳定性，使得这一类化合物具有特殊的生物活性 ${ }^{[2]}$. 发展简单有效的三氟甲基硫化合物合成方法具有重要 的学术和应用价值. 传统的三氟甲基硫化合物的合成一 般是通过氟卤交换 ${ }^{[3]}$, 以及含硫化合物如硫醚或硫醇的 三氟甲基化反应来实现的 ${ }^{[4]}$. 近年来, 许多研究小组致 力于新型三氟甲硫基化试剂的开发, 以实现多种类有机
分子的直接三氟甲硫基化反应 ${ }^{[5,6]}$, 例如：2011 年, Buchwald 研究小组 ${ }^{[7]}$ 使用 $\mathrm{AgSCF}_{3}$ 作为三氟甲硫基化试 剂首次实现了芳基溴代物的三氟甲硫基化转化; 卿风翎 小组 ${ }^{[8]}$ 则利用 $\mathrm{TMSCF}_{3}$ 和单质硫作为三氟甲硫基化试 剂，实现了芳基嗍酸的三氟甲硫基化反应；2012 年, Vicic 课题组 ${ }^{[9]}$ 使用 $\left[\mathrm{NMe}_{4}\right]\left[\mathrm{SCF}_{3}\right]$ 作为“ $\mathrm{SCF}_{3}$ ”源, 在镍催 化下与芳基碘(溴)发生交叉偶联也实现了三氟甲硫基方 向化合物的合成; 沈其龙和 Rueping 小组 ${ }^{[10]}$ 分别使用三 氟甲基次磺酸酯和 $N$-三氟甲硫基丁二酰亚胺作为三氟 甲硫基化试剂, 在铜催化下也实现了苯硼酸的三氟甲硫 基化反应; 最近, Schoenebeck 小组 ${ }^{[1]}$ 采用 $\left[\mathrm{NMe}_{4}\right]\left[\mathrm{SCF}_{3}\right]$

\footnotetext{
* Corresponding author. E-mail: xswang77@ustc.edu.cn

Received July 27, 2018; revised October 25, 2018; published online November 11, 2018.

Dedicated to Professor Qingyun Chen on the occasion of his 90th birthday.

Project supported by the National Natural Science Foundation of China (Nos. 21602213, 21522208).

国家自然科学基金(Nos. 21602213, 21522208)资助项目.

$\dagger$ 共同第一作者(These authors contributed equally to this work).
} 
作为三氟甲硫基化试剂, 在镍催化完成了与相对惰性的 芳基氯化物的交叉偶联反应.

我们小组一直致力于通过自由基途径进行的直接 三氟甲硫基化反应的研究. 2014 年 ${ }^{[12]}$, 首次开发了 $\mathrm{AgSCF}_{3} / \mathrm{K}_{2} \mathrm{~S}_{2} \mathrm{O}_{8}$ 体系, 通过三氟甲硫基自由基的温和引 发, 实现了三氟甲硫基羟吲哚的方便合成; 2016 年 ${ }^{[13]}$, 使用 $\mathrm{AgSCF}_{3} / \mathrm{K}_{2} \mathrm{~S}_{2} \mathrm{O}_{8} / \mathrm{I}_{2}$ 体系, 在温和条件下引发产生三 氟甲硫基自由基, 顺利合成了三氟甲硫基吲哚类化合 物. 烯烃的自由基含氟官能化反应是合成含氟化合物的 重要手段 ${ }^{[14]}$, 基于碳碳双键的反应特点, 烯烃可以通过 自由基途径一步引入两个官能团, 具有高度的步骤经济 性. 传统的 $\alpha$-三氟甲硫基苯乙酮合成方法有两种: 一是 $\alpha$-溴代苯乙酮与三氟甲硫基化试剂的取代反应 ${ }^{[15]}$; 二是 通过苯乙酮类化合物的 $\alpha$-位脱氢烯醇化, 进而与三氟甲 硫基化试剂进行作用, 实现 $\alpha$-三氟甲硫基苯乙酮的合 成 ${ }^{[16]}$. 最近, Singh 课题组 ${ }^{[17]}$ 利用光催化剂通过苯乙烯 氧化双官能化反应实现该类化合物的合成. 本工作使用 $\mathrm{AgSCF}_{3} / \mathrm{K}_{2} \mathrm{~S}_{2} \mathrm{O}_{8}$ 体系, 在无需外加催化剂的条件下, 通 过自由基启动的烯烃双官能化反应, 实现了不同取代的 $\alpha$-三氟甲硫基苯乙酮的合成.

\section{1 结果与讨论}

\section{1 反应条件优化}

选用苯乙烯 $1 \mathrm{a}$ 作为模板底物, 以 $\mathrm{AgSCF}_{3}$ 作为三氟 甲硫基源, $\mathrm{K}_{2} \mathrm{~S}_{2} \mathrm{O}_{8}$ 作为氧化剂, 在 $60{ }^{\circ} \mathrm{C}$ 下应 $24 \mathrm{~h}$, 我 们以 $16 \%$ 的核磁收率得到三氟甲硫基化/氧化产物 $2 \mathbf{a}$. 对氧化剂进行考察时发现, 当使用 $\mathrm{Na}_{2} \mathrm{~S}_{2} \mathrm{O}_{8}$ 作为氧化
剂时, 收率略有下降，而当氧化剂变为 $\mathrm{PhI}(\mathrm{OAc})_{2}$ 和 NSFI 时, 几乎不发生反应(表 1, Entries 1 4). 随后, 我 们尝试加入一些过渡金属催化剂, 发现常用的铜和银等 催化剂的加入会抑制反应的发生(表 1, Entries 5,6). 而 当将磷酸氢二钾作为碱加至反应体系中, 产率略有提 高, 达到 22\%(表 1, Entry 7). 在对反应体系的溶剂进行 篮选时发现, 当使用 $\mathrm{DMSO} / \mathrm{CHCl}_{3}(V / V=2 / 1,3.0 \mathrm{~mL})$ 时，可以得到 $49 \%$ 的产率(表 1 , Entries $8 \sim 11$ ). 而在氧气 氛围下，反应的收率可以提升至 $68 \%$ (表 1, Entry 12). 最 后, 将氧化剂 $\mathrm{K}_{2} \mathrm{~S}_{2} \mathrm{O}_{8}$ 用量降低至 1.5 equiv., 反应的收率 略有提高(69\%, 表 1, Entry 13).

\section{2 反应底物拓展}

在最优的反应条件下, 我们接下来对反应的底物适 用性进行了研究(表 2). 首先考察了供电子基团甲氧基 在不同位置时的反应情况，反应都以中等到良好的收率 得到目标产物 $\mathbf{2 b} \sim \mathbf{2 d}$ (表 2); 对于甲基取代的苯乙烯, 可以得到中等收率 (表 $2 ， \mathbf{2} \mathbf{e} \sim 2 \mathrm{~g}$ ). 当取代基团为卤素原 子时, 可以以中等到良好的收率得到目标产物(表 2, $\mathbf{2 h} \sim \mathbf{2 m}$ ), 尤其是当取代基团为溴原子时，可以得到中 等偏上的收率, 这也为进一步衍生化提供了可能. 随后, 我们考察了吸电子基团对反应活性的影响: 如氧基、酯 基、三氟甲基取代时，都可以得到 50\%以上的分离收率 (表 2, 2n 2q). 综上所述, 无论是吸电子基团还是给电 子基团取代的苯乙烯，都可以得到中等到良好的收率. 苯环上不同位置的取代基对反应略有影响. 其中, 苯环 上存在对位 $(2 \mathrm{~b}, 2 \mathrm{e}, 2 \mathrm{~h}, 2 \mathrm{j}, 2 \mathrm{l}, 2 \mathrm{n}, 2 \mathrm{o}, 2 \mathrm{p})$ 或间位取代基 $(2 \mathrm{c}, 2 \mathrm{f}, 2 \mathrm{i}, 2 \mathrm{k}, 2 \mathrm{~m}, 2 \mathrm{q})$ ), 都能够以中等到良好的收率得

表 1 反应条件优化 ${ }^{a}$

Table 1 Optimization of reaction conditions

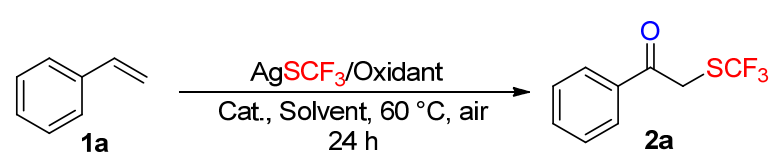

\begin{tabular}{|c|c|c|c|c|}
\hline Entry & Oxidant (equiv.) & Cat. (mol\%) & Solvent (mL) & Yield/\% \\
\hline 1 & $\mathrm{~K}_{2} \mathrm{~S}_{2} \mathrm{O}_{8}(3.0)$ & None & $\mathrm{MeCN}(1.0)$ & 16 \\
\hline 2 & $\mathrm{~K}_{2} \mathrm{~S}_{2} \mathrm{O}_{8}(3.0)$ & None & $\operatorname{MeCN}(1.0)$ & 14 \\
\hline 3 & $\mathrm{PhI}(\mathrm{OAc})_{2}(3.0)$ & None & $\mathrm{MeCN}(1.0)$ & Trace \\
\hline 4 & NFSI (3.0) & None & $\mathrm{MeCN}(1.0)$ & Trace \\
\hline 5 & $\mathrm{~K}_{2} \mathrm{~S}_{2} \mathrm{O}_{8}(3.0)$ & $\mathrm{CuI}(20)$ & $\mathrm{MeCN}(1.0)$ & Trace \\
\hline 6 & $\mathrm{~K}_{2} \mathrm{~S}_{2} \mathrm{O}_{8}(3.0)$ & $\mathrm{AgNO}_{3}(20)$ & $\mathrm{MeCN}(1.0)$ & 0 \\
\hline $7^{b}$ & $\mathrm{~K}_{2} \mathrm{~S}_{2} \mathrm{O}_{8}(3.0)$ & None & $\operatorname{MeCN}(1.0)$ & 22 \\
\hline 8 & $\mathrm{~K}_{2} \mathrm{~S}_{2} \mathrm{O}_{8}(3.0)$ & None & $\operatorname{DMF}(1.0)$ & 0 \\
\hline 9 & $\mathrm{~K}_{2} \mathrm{~S}_{2} \mathrm{O}_{8}(3.0)$ & None & DMSO (1.0) & 20 \\
\hline 10 & $\mathrm{~K}_{2} \mathrm{~S}_{2} \mathrm{O}_{8}(3.0)$ & None & $\mathrm{DMSO} / \mathrm{CHCl}_{3}(V: V=1: 1,1.0)$ & 27 \\
\hline 11 & $\mathrm{~K}_{2} \mathrm{~S}_{2} \mathrm{O}_{8}(3.0)$ & None & $\mathrm{DMSO} / \mathrm{CHCl}_{3}(V: V=2: 1,3.0)$ & 49 \\
\hline $12^{c}$ & $\mathrm{~K}_{2} \mathrm{~S}_{2} \mathrm{O}_{8}(3.0)$ & None & $\mathrm{DMSO} / \mathrm{CHCl}_{3}(V: V=2: 1,3.0)$ & 68 \\
\hline $13^{c}$ & $\mathrm{~K}_{2} \mathrm{~S}_{2} \mathrm{O}_{8}(1.5)$ & None & $\mathrm{DMSO} / \mathrm{CHCl}_{3}(V: V=2: 1,3.0)$ & $69(70)$ \\
\hline
\end{tabular}

${ }^{a}$ 1a $(0.1 \mathrm{mmol}), \mathrm{AgSCF}_{3}(0.15 \mathrm{mmol}, 1.5$ equiv. $), \mathrm{K}_{2} \mathrm{~S}_{2} \mathrm{O}_{8}$, slovent, $60{ }^{\circ} \mathrm{C}, 24 \mathrm{~h} .{ }^{19} \mathrm{~F}$ NMR yields with $\mathrm{PhCF}_{3}(0.1 \mathrm{mmol})$ as an internal standard. ${ }^{b} \mathrm{~K}_{2} \mathrm{HPO}_{4}(0.15$ mmol) was added. ${ }^{c}$ Reaction was performed under $\mathrm{O}_{2}$ atmosphere. ${ }^{d}$ Isolated yields were displayed in parentheses. 
表 2 反应底物拓展 ${ }^{a}$

Table 2 Substrate scope of different styrenes
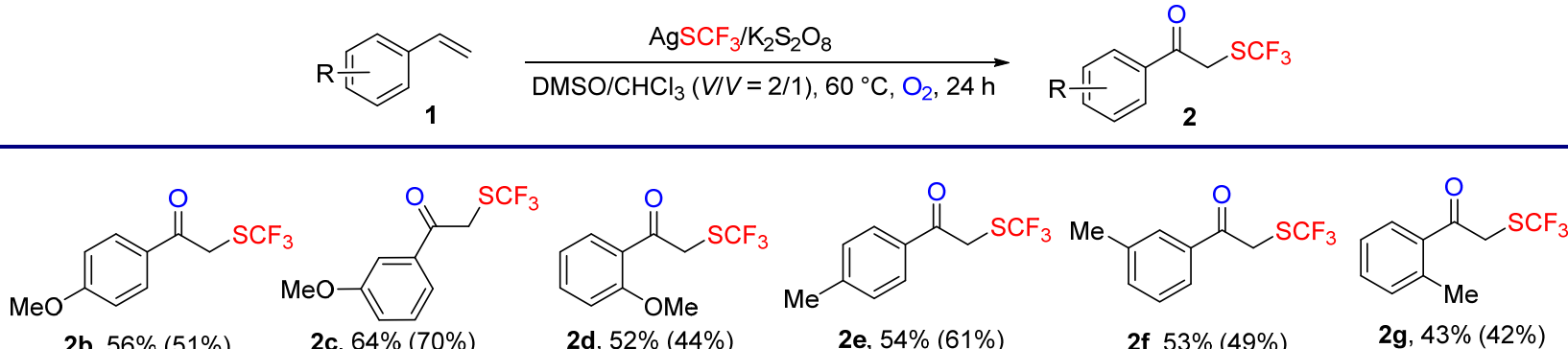

2b, $56 \%(51 \%)$

2c, $64 \%(70 \%)$

$2 d, 52 \%(44 \%)$

$2 e, 54 \%(61 \%)$

2f, $53 \%(49 \%)$

2g, $43 \%(42 \%)$<smiles>O=C(CSc1ccccc1)c1ccc(Cl)cc1</smiles><smiles>O=C(C[AsH2](F)(F)F)c1cccc(Cl)c1</smiles>

2h, $56 \%$ (53\%)<smiles>O=C(C[AsH2]C(F)(F)F)c1cccc(F)c1</smiles>

$2 \mathrm{~m}, 63 \%(51 \%)$ 2i, $60 \%(59 \%)$<smiles>N#Cc1ccc(C(=O)C[Ge]F)cc1</smiles>

2n, $60 \%(51 \%)$<smiles>O=C(C[Ge](F)(F)F)c1ccc(Br)cc1</smiles>

2j, $64 \%(62 \%)$<smiles>COC(=O)c1ccc(C(=O)CC(F)(F)F)cc1</smiles>

2o, $62 \%(66 \%)$<smiles>O=C(C[AsH2](F)(F)F)c1cccc(Br)c1</smiles>

2k, $65 \%(61 \%)$<smiles>O=C(CSc1ccccc1)c1ccc(F)cc1</smiles>

$21,62 \%(60 \%)$

${ }^{a} \mathbf{1}(0.1 \mathrm{mmol}), \mathrm{AgSCF}_{3}(0.15 \mathrm{mmol}, 1.5$ equiv. $), \mathrm{K}_{2} \mathrm{~S}_{2} \mathrm{O}_{8}(0.15 \mathrm{mmol}, 1.5$ equiv. $), \mathrm{DMSO}(2 \mathrm{~mL}), \mathrm{CHCl}_{3}(1 \mathrm{~mL}), 60{ }^{\circ} \mathrm{C}, \mathrm{O}_{2}, 24 \mathrm{~h} .{ }^{19} \mathrm{~F} \mathrm{NMR} \mathrm{yields} \mathrm{with} \mathrm{PhCF}_{3}(0.1$ $\mathrm{mmol})$ as an internal standard. Isolated yields were displayed in parentheses.

到对应的三氟甲硫基化产物. 但当苯环邻位有取代基时 $(2 \mathrm{~d}, \mathbf{2 g})$, 反应通常收率有所降低, 这可能是由于位阻作 用阻碍了反应的进行.

\section{3 反应机理研究}

为了验证反应机理, 我们在标准反应体系中分别加 入 0.5 equiv.和 1.0 equiv. 的自由基捕获剂 2,2,6,6-四甲基 哌啶氮氧化物(TEMPO)进行反应尝试. 发现当加入 0.5 equiv. 的 TEMPO 时, 仍然可以得到 $51 \%$ 的目标产物 $\mathbf{2 a}$, 而增加 TEMPO 的用量到 1.0 equiv. 时, 则没有检测到目 标产物的生成(表 3, Entries 1, 2). 在体系中加入 1.0 equiv. 的对二硝基苯时, 也不能得到标准产物 $\mathbf{2} \mathbf{a}$ (表 3 , Entry 3). 这些结果说明了该反应可能是通过自由基历 程进行的. 随后, 为了确定产物中氧的来源, 在氮气条 件下进行了反应的尝试，没有得到目标产物 $\mathbf{2 a}$ (表 3 , Entry 4). 这一结果说明产物中的氧原子可能来源于氧 气 $^{[17]}$.

结合以上验证实验的结果和现有文献报道 ${ }^{[17]}$, 我 们提出以下可能的机理: 首先三氟甲硫基银试剂在过硫 酸钾作用下产生三氟甲硫基自由基, 该自由基进攻苯乙 烯(1a)得到苄基自由基中间体 $\mathbf{A}$, 氧气捕获苠基自由基 产生过氧自由基 $\mathbf{B}$, 进一步生成中间体 $\mathbf{C}$, 随后氧化得 到氧化三氟甲硫基化产物 $\alpha$-三氟甲硫基苯乙酮 $2 \mathbf{a}$ (Scheme 1).

\section{2 结论}

使用我们小组发展的 $\mathrm{AgSCF}_{3} / \mathrm{K}_{2} \mathrm{~S}_{2} \mathrm{O}_{8}$ 自由基三氟甲
表 3 机理研究

Table 3 Mechanism research
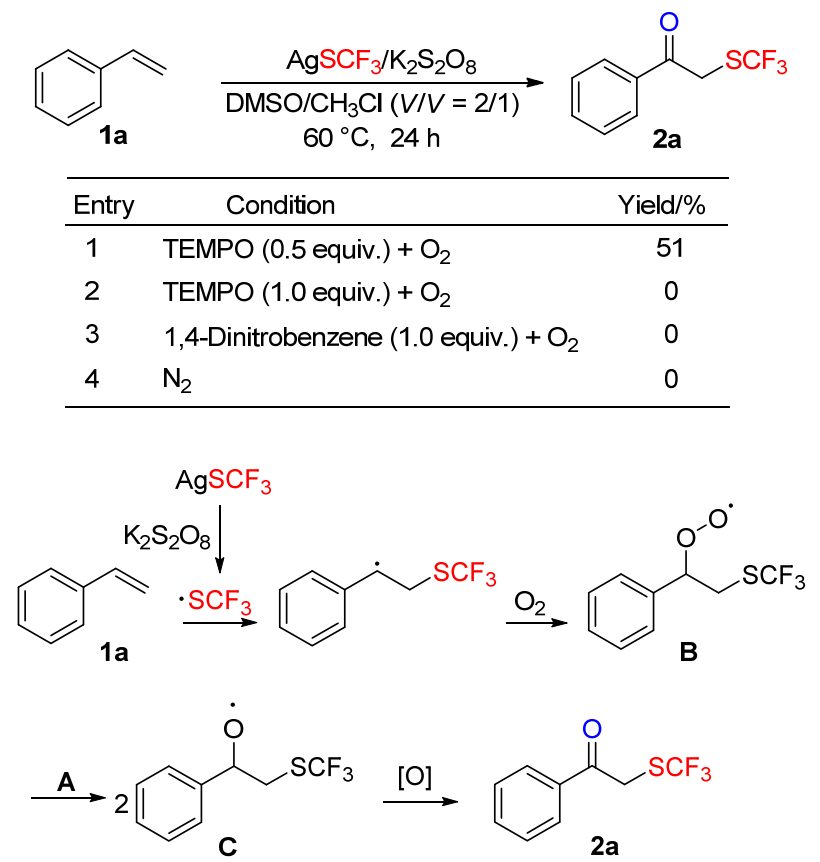

图式 1 可能的反应机理

Scheme 1 Possible mechanism

硫基化体系，发展了苯乙烯的三氟甲硫基化/氧化串联 反应，通过步骤经济的双官能团化反应实现了三氟甲硫 基化苯乙酮的高效合成. 该反应以稳定的三氟甲硫基银 为三氟甲硫基源, 便宜易得的 $\mathrm{K}_{2} \mathrm{~S}_{2} \mathrm{O}_{8}$ 为氧化剂, 无需外 加催化剂，以中等到良好的收率实现一系列不同取代的 
苯乙烯的氧化三氟甲硫基化转化.

\section{3 实验部分}

\section{1 仪器与试剂}

所有化合物的氢谱、碳谱和氟谱均采用 Bruker-400 (氢谱 $400 \mathrm{MHz}$ 、碳谱 $100 \mathrm{MHz}$ 、氟谱 $376 \mathrm{MHz}$ ) 傅里 叶转换核磁共振仪测定, 分别使用四甲基硅烷或者三氯 氟甲烷作为内标. 苯乙烯, 4-甲基苯乙烯, 4-甲氧基苯乙 烯从 Sinopharm Chemical 公司购买, 重蒸后使用. $\mathrm{K}_{2} \mathrm{~S}_{2} \mathrm{O}_{8}$ 从 Sinopharm Chemical 公司购买并且直接使用. $\mathrm{AgSCF}_{3}$ 是按照 Buchwald 小组报道的方法制备 ${ }^{[7]}$. 其他 苯乙烯衍生物按照文献报道制备(详见辅助材料).

\section{2 实验方法}

将三氟甲硫基银 $(31.4 \mathrm{mg}, 0.15 \mathrm{mmol}$ )和过硫酸钾 (40.6 mg, $0.15 \mathrm{mmol}$ ) 依次加入到预先干燥过的 $50 \mathrm{~mL}$ 反 应管中. 在氧气氛围下加入苯乙烯 $(\mathbf{1 a}, 0.1 \mathrm{mmol})$, 二甲 亚砜 $(2.0 \mathrm{~mL})$, 氯仿 $(1.0 \mathrm{~mL})$, 将该反应管放置在预先加 热的 $60{ }^{\circ} \mathrm{C}$, 反应 $24 \mathrm{~h}$ 后, 冷却至室温, 使用乙酸乙酯 萃取三次, 无水硫酸钠干燥, 浓缩, 柱层析分离 [洗脱 剂: $V($ 石油醚 $): V($ 乙酸乙酯 $)=10 ： 1$ ] 得到目标产物 2 .

\section{3 化合物结构数据}

报道的所有化合物表征数据均与参考文献 $[15 \sim 18]$ 一致.

1-苯基-2-三氟甲硫基-1-乙酮(2a) ${ }^{[15 a]}$ : ${ }^{1} \mathrm{H}$ NMR (400 $\left.\mathrm{MHz}, \mathrm{CDCl}_{3}\right) \delta: 7.97 \sim 7.95(\mathrm{~m}, 2 \mathrm{H}), 7.64(\mathrm{t}, J=7.6 \mathrm{~Hz}$, $1 \mathrm{H}), 7.52(\mathrm{t}, J=7.6 \mathrm{~Hz}, 2 \mathrm{H}), 4.53(\mathrm{~s}, 2 \mathrm{H}) ;{ }^{13} \mathrm{C}$ NMR $(101$ $\mathrm{MHz}, \mathrm{CDCl}_{3}$ ) $\delta: 192.0$ (s), 134.7 (s), 134.3 (s), 130.8 (q, $J=307 \mathrm{~Hz}$ ), 129.0 (s), 128.4 (s), 38.4 (q, $J=2.0 \mathrm{~Hz}$ ); ${ }^{19} \mathrm{~F}$ NMR $\left(376 \mathrm{MHz}, \mathrm{CDCl}_{3}\right) \delta:-41.4(\mathrm{~s}, 3 \mathrm{~F})$.

1-(4-甲氧基苯基)-2-三氟甲硫基-1-乙酮 $(\mathbf{2 b})^{[15 \mathrm{a}]}$ : ${ }^{1} \mathrm{H}$ NMR (400 MHz, $\left.\mathrm{CDCl}_{3}\right) \delta: 7.93(\mathrm{~d}, J=8.8 \mathrm{~Hz}, 2 \mathrm{H}), 6.96$ (d, $J=8.8 \mathrm{~Hz}, 2 \mathrm{H}), 4.48$ (s, 2H), 3.89 (s, 3H); ${ }^{13} \mathrm{C} \mathrm{NMR}$ $\left(101 \mathrm{MHz}, \mathrm{CDCl}_{3}\right) \delta: 190.5$ (s), 164.5 (s), 130.9 (q, $J=$ $307.3 \mathrm{~Hz}$ ), 130.9 (s), 127.8 (s), 114.3 (s), 55.7 (s), 38.3 (q, $J=1.9 \mathrm{~Hz}) ;{ }^{19} \mathrm{~F} \mathrm{NMR}\left(376 \mathrm{MHz}, \mathrm{CDCl}_{3}\right) \delta:-41.4(\mathrm{~s}$, $3 \mathrm{~F})$.

1-(3-甲氧基苯基)-2-三氟甲硫基-1-乙酮 $(2 \mathrm{c})^{[15 \mathrm{a}]}{ }^{1} \mathrm{H}$ NMR (400 MHz, $\left.\mathrm{CDCl}_{3}\right) \delta: 7.51(\mathrm{~d}, J=7.6 \mathrm{~Hz}, 1 \mathrm{H}), 7.48$ (s, 1H), $7.42(\mathrm{td}, J=7.6,1.2 \mathrm{~Hz}, 1 \mathrm{H}), 7.18(\mathrm{~d}, J=8.0 \mathrm{~Hz}$, 1H), 4.51 (s, 2H), $3.87(\mathrm{~s}, 3 \mathrm{H}) ;{ }^{13} \mathrm{C} \mathrm{NMR}(101 \mathrm{MHz}$, $\mathrm{CDCl}_{3}$ ) $\delta: 191.8$ (s), 160.1 (s), 136.0 (s), 130.7 (q, $J=307$ Hz), 130.0 (s), 121.0 (s), 120.7 (s), 112.6 (s), 55.5 (s), 38.4 $(\mathrm{q}, J=2 \mathrm{~Hz}) ;{ }^{19} \mathrm{~F}$ NMR $\left(376 \mathrm{MHz}, \mathrm{CDCl}_{3}\right) \delta$ : -41.5 (s, 3F).

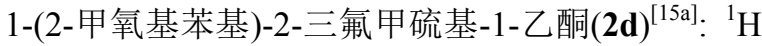
NMR (400 MHz, $\left.\mathrm{CDCl}_{3}\right) \delta: 7.88(\mathrm{~d}, J=7.6 \mathrm{~Hz}, 1 \mathrm{H}), 7.50$ $(\mathrm{t}, J=7.2 \mathrm{~Hz}, 1 \mathrm{H}), 7.08 \sim 7.00(\mathrm{~m}, 2 \mathrm{H}), 4.43(\mathrm{~s}, 2 \mathrm{H}), 3.95$ (s, 3H); ${ }^{13} \mathrm{C}$ NMR (101 MHz, $\left.\mathrm{CDCl}_{3}\right) \delta: 193.2$ (s), 159.0 (s), 135.2 (s), 131.4 (s), 130.9 (q, J=307 Hz), 124.7 (s), 121.1 (s), 111.6 (s), 55.7 (s), 42.5 (q, $J=2.0 \mathrm{~Hz}) ;{ }^{19} \mathrm{~F}$ NMR $\left(376 \mathrm{MHz} \mathrm{CDCl}_{3}\right) \delta$ : $-41.6(\mathrm{~s}, 3 \mathrm{~F})$.

1-(4-甲基苯基)-2-三氟甲硫基-1-乙酮 $(2 \mathrm{e})^{[15 \mathrm{a}]}$ : ${ }^{1} \mathrm{H}$ NMR (400 MHz, $\left.\mathrm{CDCl}_{3}\right) \delta: 7.85(\mathrm{~d}, J=8.0 \mathrm{~Hz}, 2 \mathrm{H}), 7.30$ $(\mathrm{d}, J=8.0 \mathrm{~Hz}, 2 \mathrm{H}), 4.50(\mathrm{~s}, 2 \mathrm{H}), 2.44(\mathrm{~s}, 3 \mathrm{H}) ;{ }^{13} \mathrm{C} \mathrm{NMR}$ (101 MHz, $\mathrm{CDCl}_{3}$ ) $\delta: 191.2$ (s), 145.4 (s), 132.2 (s), 130.7 (q, $J=307.0 \mathrm{~Hz}), 129.6$ (s), $128.5(\mathrm{~s}), 38.4$ (q, $J=2.0 \mathrm{~Hz}$ ), $21.8(\mathrm{~s}) ;{ }^{19} \mathrm{~F}$ NMR $\left(376 \mathrm{MHz}, \mathrm{CDCl}_{3}\right) \delta:-41.4(\mathrm{~s}, 3 \mathrm{~F})$.

1-(3-甲基苯基)-2-三氟甲硫基-1-乙酮 $(2 \mathbf{f})^{[15 c]}:{ }^{1} \mathrm{H}$ NMR (400 MHz, $\left.\mathrm{CDCl}_{3}\right) \delta: 7.75 \sim 7.73(\mathrm{~m}, 2 \mathrm{H}), 7.45(\mathrm{~d}$, $J=7.6 \mathrm{~Hz}, 1 \mathrm{H}), 7.39$ (t, $J=7.6 \mathrm{~Hz}, 1 \mathrm{H}), 4.50(\mathrm{~s}, 2 \mathrm{H}), 2.43$ (s, 3H); ${ }^{13} \mathrm{C}$ NMR (101 MHz, $\left.\mathrm{CDCl}_{3}\right) \delta: 192.1$ (s), 138.9 (s), 135.0 (s), 134.7 (s), 130.8 (q, $J=307.0 \mathrm{~Hz}$ ), 128.9 (s), 128.8(s), $125.6(\mathrm{~s}), 38.5$ (q, $J=2.0 \mathrm{~Hz}), 21.3(\mathrm{~s}) ;{ }^{19} \mathrm{~F}$ NMR $\left(376 \mathrm{MHz}, \mathrm{CDCl}_{3}\right) \delta$ : $-41.4(\mathrm{~s}, 3 \mathrm{~F})$.

1-(2-甲基苯基)-2-三氟甲硫基-1-乙酮 $(2 \mathrm{~g})^{[15 \mathrm{c}]}$ : ${ }^{1} \mathrm{H}$ NMR (400 MHz, $\left.\mathrm{CDCl}_{3}\right) \delta: 7.67$ (dd, $\left.J=7.6,0.8 \mathrm{~Hz}, 1 \mathrm{H}\right)$, $7.48 \sim 7.44(\mathrm{~m}, 1 \mathrm{H}), 7.33 \sim 7.30(\mathrm{~m}, 2 \mathrm{H}), 4.46(\mathrm{~s}, 2 \mathrm{H})$, $2.54(\mathrm{~s}, 3 \mathrm{H}) ;{ }^{13} \mathrm{C}$ NMR (101 MHz, $\left.\mathrm{CDCl}_{3}\right) \delta: 194.7,139.8$, 134.6, 132.7, 132.5, 130.7 (q, $J=307.0 \mathrm{~Hz}$ ), 129.1, 126.0, 40.4 (q, $J=2.0 \mathrm{~Hz}), 21.6 ;{ }^{19} \mathrm{~F}$ NMR $\left(376 \mathrm{MHz}, \mathrm{CDCl}_{3}\right)$ $\delta:-41.4(\mathrm{~s}, 3 \mathrm{~F})$.

1-(4-氯苯基)-2-三氟甲硫基-1-乙酮 (2h) ${ }^{[15 a]}:{ }^{1} \mathrm{H}$ NMR (400 MHz, $\mathrm{CDCl}_{3}$ ) $\delta: 7.91(\mathrm{~d}, J=8.4 \mathrm{~Hz}, 2 \mathrm{H}), 7.49$ (d, $J=8.8 \mathrm{~Hz}, 2 \mathrm{H}), 4.47(\mathrm{~s}, 2 \mathrm{H}) ;{ }^{13} \mathrm{C} \mathrm{NMR}(101 \mathrm{MHz}$, $\mathrm{CDCl}_{3}$ ) $\delta: 190.9$ (s), 140.9 (s), 133.0 (s), 130.5 (q, $J=$ $308.0 \mathrm{~Hz}$ ), 129.8 (s), 129.4 (s), 38.2 (q, $J=2.0 \mathrm{~Hz}) ;{ }^{19} \mathrm{~F}$ NMR $\left(376 \mathrm{MHz}, \mathrm{CDCl}_{3}\right) \delta$ : $-41.4(\mathrm{~s}, 3 \mathrm{~F})$.

1-(3-氯苯基)-2-三氟甲硫基-1-乙酮 $(\mathbf{2 i})^{[15 c]}$ : ${ }^{1} \mathrm{H}$ NMR $\left(400 \mathrm{MHz}, \mathrm{CDCl}_{3}\right) \delta: 7.93(\mathrm{~d}, J=2.0 \mathrm{~Hz}, 1 \mathrm{H}), 7.85 \sim 7.82$ $(\mathrm{m}, 1 \mathrm{H}), 7.63 \sim 7.61(\mathrm{~m}, 1 \mathrm{H}), 7.47(\mathrm{t}, J=8.0 \mathrm{~Hz}, 1 \mathrm{H}), 4.48$ (s, 2H); ${ }^{13} \mathrm{C}$ NMR (101 MHz, $\left.\mathrm{CDCl}_{3}\right) \delta: 190.8$ (s), 136.1 (s), 135.4 (s), 134.2 (s), 130.5 (q, $J=308.1 \mathrm{~Hz}$ ), 130.3 (s), 128.5 (s), 126.5 (s), 38.2 (q, $J=2.0 \mathrm{~Hz}) ;{ }^{19} \mathrm{~F}$ NMR (376 $\left.\mathrm{MHz}, \mathrm{CDCl}_{3}\right) \delta:-41.4(\mathrm{~s}, 3 \mathrm{~F})$.

1-(4-溴苯基)-2-三氟甲硫基-1-乙酮 $(\mathbf{2} \mathbf{j})^{[15 a]}$ : ${ }^{1} \mathrm{H}$ NMR $\left(400 \mathrm{MHz}, \mathrm{CDCl}_{3}\right) \delta: 7.81(\mathrm{~d}, J=8.4 \mathrm{~Hz}, 2 \mathrm{H}), 7.65(\mathrm{~d}, J=$ $8.4 \mathrm{~Hz}, 2 \mathrm{H}), 4.47(\mathrm{~s}, 2 \mathrm{H}) ;{ }^{13} \mathrm{C} \mathrm{NMR}\left(101 \mathrm{MHz}, \mathrm{CDCl}_{3}\right) \delta$ : 191.2 (s), 133.5 (s), 132.5 (s), 130.7 (q, $J=306.7$ Hz), 130.0 (s), 129.8 (s), 38.2 (q, $J=1.9 \mathrm{~Hz}) ;{ }^{19} \mathrm{~F}$ NMR (376 
$\left.\mathrm{MHz}, \mathrm{CDCl}_{3}\right) \delta:-41.4(\mathrm{~s}, 3 \mathrm{~F})$.

1-(3- 溴苯基)-2-三氟甲硫基-1-乙酮 $(\mathbf{2 k})^{[15 c]}$ : ${ }^{1} \mathrm{H}$ NMR (400 MHz, $\left.\mathrm{CDCl}_{3}\right) \delta: 8.08(\mathrm{t}, J=1.6 \mathrm{~Hz}, 1 \mathrm{H})$, $7.89 \sim 7.86(\mathrm{~m}, 1 \mathrm{H}), 7.78 \sim 7.75(\mathrm{~m}, 1 \mathrm{H}), 7.40(\mathrm{t}, J=8.0$ $\mathrm{Hz}, 1 \mathrm{H}), 4.48(\mathrm{~s}, 2 \mathrm{H}) .{ }^{13} \mathrm{C} \mathrm{NMR}\left(101 \mathrm{MHz}, \mathrm{CDCl}_{3}\right) \delta$ : 190.7 (s), 137.1 (s), 136.3 (s), 131.4 (s), 130.5 (q, $J=308.1$ $\mathrm{Hz}), 130.5$ (s), 126.9 (s), 123.3 (s), 38.2 (q, $J=2.0 \mathrm{~Hz}$ ); ${ }^{19} \mathrm{~F}$ NMR $\left(376 \mathrm{MHz}, \mathrm{CDCl}_{3}\right) \delta$ : $-41.4(\mathrm{~s}, 3 \mathrm{~F})$.

1-(4-氟苯基)-2-三氟甲硫基-1-乙酮(2l $)^{[15 a]}$ : ${ }^{1} \mathrm{H}$ NMR $\left(400 \mathrm{MHz}, \mathrm{CDCl}_{3}\right) \delta: 8.02 \sim 7.98(\mathrm{~m}, 2 \mathrm{H}), 7.19(\mathrm{t}, J=8.4$ $\mathrm{Hz}, 2 \mathrm{H}), 4.49(\mathrm{~s}, 2 \mathrm{H}) ;{ }^{13} \mathrm{C} \mathrm{NMR}\left(101 \mathrm{MHz}, \mathrm{CDCl}_{3}\right) \delta$ : 190.4 (s), 166.4 (d, $J=258.6 \mathrm{~Hz}), 131.2$ (d, $J=10.1 \mathrm{~Hz})$, 130.6 (q, $J=307.0 \mathrm{~Hz}), 116.2$ (d, $J=22.2 \mathrm{~Hz}), 38.2$ (q, $J=$ $2.0 \mathrm{~Hz}) ;{ }^{19} \mathrm{~F}$ NMR $\left(376 \mathrm{MHz}, \mathrm{CDCl}_{3}\right) \delta:-41.5(\mathrm{~s}$, $3 \mathrm{~F}),-102.6(\mathrm{~s}, 1 \mathrm{~F})$.

1-(3-氟苯基)-2-三氟甲硫基-1-乙酮 $(\mathbf{2 m})^{[18]}$ : ${ }^{1} \mathrm{H}$ NMR $\left(400 \mathrm{MHz}, \mathrm{CDCl}_{3}\right) \delta: 7.75 \sim 7.72(\mathrm{~m}, 1 \mathrm{H}), 7.67 \sim$ $7.64(\mathrm{~m}, 1 \mathrm{H}), 7.54 \sim 7.49(\mathrm{~m}, 1 \mathrm{H}), 7.38 \sim 7.33(\mathrm{~m}, 1 \mathrm{H})$, 4.49 (s, 2H); ${ }^{13} \mathrm{C}$ NMR (101 MHz, $\left.\mathrm{CDCl}_{3}\right) \delta: 190.8$ (d, $J=$ $2.0 \mathrm{~Hz}), 162.9$ (d, $J=49.5 \mathrm{~Hz}), 136.6$ (d, $J=6.0 \mathrm{~Hz}), 130.8$ $(\mathrm{q}, J=8.1 \mathrm{~Hz}), 130.5(\mathrm{q}, J=308.1 \mathrm{~Hz}), 124.2(\mathrm{~d}, J=4.0$ $\mathrm{Hz}), 121.4$ (d, $J=22.2 \mathrm{~Hz}), 115.2$ (d, $J=22.2 \mathrm{~Hz}), 38.2$ (q, $J=2.0 \mathrm{~Hz}) ;{ }^{19} \mathrm{~F}$ NMR $\left(376 \mathrm{MHz}, \mathrm{CDCl}_{3}\right) \delta:-41.4(\mathrm{~s}$, $3 \mathrm{~F}),-111.1(\mathrm{~s}, 1 \mathrm{~F})$.

1-(4-㲵基苯基)-2-三氟甲硫基-1-乙酮 $(\mathbf{2 n})^{[15 \mathrm{a}]}{ }^{1} \mathrm{H}$ NMR (400 MHz, $\left.\mathrm{CDCl}_{3}\right) \delta: 8.06(\mathrm{~d}, J=8.4 \mathrm{~Hz}, 2 \mathrm{H}), 7.83$ $(\mathrm{d}, J=8.8 \mathrm{~Hz}, 2 \mathrm{H}), 4.49(\mathrm{~s}, 2 \mathrm{H}) ;{ }^{13} \mathrm{C}$ NMR $(101 \mathrm{MHz}$, $\left.\mathrm{CDCl}_{3}\right) \delta: 190.9$ (s), 137.5 (s), 132.8 (s), 130.3 (q, $J=$ $308.1 \mathrm{~Hz}$ ), 128.8 (s), 117.5 (s), 38.0 (q, $J=2.0 \mathrm{~Hz}$ ); ${ }^{19} \mathrm{~F}$ NMR $\left(376 \mathrm{MHz}, \mathrm{CDCl}_{3}\right) \delta$ : $-41.4(\mathrm{~s}, 3 \mathrm{~F})$.

4-(2-(三氟甲硫基))乙酰基苯甲酸甲酯 $(20){ }^{[15 a]}$ : ${ }^{1} \mathrm{H}$ NMR (400 MHz, $\left.\mathrm{CDCl}_{3}\right) \delta: 8.16(\mathrm{~d}, J=8.4 \mathrm{~Hz}, 2 \mathrm{H}), 8.01$ $(\mathrm{d}, J=8.4 \mathrm{~Hz}, 2 \mathrm{H}), 4.53$ (s, 2H), $3.96(\mathrm{~s}, 3 \mathrm{H}) ;{ }^{13} \mathrm{C}$ NMR (101 MHz, $\left.\mathrm{CDCl}_{3}\right) \delta: 191.8$ (s), 166.0 (s), 137.9 (s), 135.1 (s), 130.7 (q, $J=307.5 \mathrm{~Hz}$ ), 130.3 (s), 128.5 (s), 52.8 (s), $38.6(\mathrm{q}, J=1.9 \mathrm{~Hz}) ;{ }^{19} \mathrm{~F}$ NMR $\left(376 \mathrm{MHz}, \mathrm{CDCl}_{3}\right) \delta$ : $-41.4(\mathrm{~s}, 3 \mathrm{~F})$.

1-(4-三氟甲基苯基)-2-三氟甲硫基-1-乙酮 $(2 \mathbf{p})^{[18]}$ : ${ }^{1} \mathrm{H}$ NMR (400 MHz, $\left.\mathrm{CDCl}_{3}\right) \delta: 8.07$ (d, $\left.J=8.0 \mathrm{~Hz}, 2 \mathrm{H}\right)$, 7.79 (d, $J=8.4 \mathrm{~Hz}, 2 \mathrm{H}), 4.51$ (s, 2H); ${ }^{13} \mathrm{C}$ NMR (101 MHz, $\left.\mathrm{CDCl}_{3}\right) \delta: 191.2(\mathrm{~s}), 137.3$ (s), 135.5 (q, $J=33.3 \mathrm{~Hz}$ ), 130.4 (q, $J=307.0 \mathrm{~Hz}), 128.8$ (s), 126.1 (q, $J=4.0 \mathrm{~Hz}$ ), $123.3(\mathrm{q}, J=273.7 \mathrm{~Hz}), 38.2(\mathrm{q}, J=2.0 \mathrm{~Hz}) ;{ }^{19} \mathrm{~F}$ NMR $\left(376 \mathrm{MHz} \mathrm{CDCl}_{3}\right) \delta:-41.4(\mathrm{~s}, 3 \mathrm{~F}),-63.3(\mathrm{~s}, 3 \mathrm{~F})$.

1-(3-三氟甲基苯基)-2-三氟甲硫基-1-乙酮(2q) ${ }^{[15 c]}$.
${ }^{1} \mathrm{H}$ NMR $\left(400 \mathrm{MHz}, \mathrm{CDCl}_{3}\right) \delta: 8.2(\mathrm{~s}, 1 \mathrm{H}), 8.15(\mathrm{~d}, J=8.0$ $\mathrm{Hz}, 1 \mathrm{H}), 7.90$ (d, $J=8.0 \mathrm{~Hz}, 1 \mathrm{H}), 7.68$ (t, $J=7.6 \mathrm{~Hz}, 1 \mathrm{H})$, 4.52 (s, 2H); ${ }^{13} \mathrm{C}$ NMR (101 MHz, $\left.\mathrm{CDCl}_{3}\right) \delta: 190.8$ (s), 135.2 (s), 131.7 (q, $J=33.3 \mathrm{~Hz}), 131.5$ (s), 130.6 (q, $J=$ $4.0 \mathrm{~Hz}), 130.4$ (q, $J=308.1 \mathrm{~Hz}), \quad 129.8$ (s), 125.2 (q, $J=$ $4.0 \mathrm{~Hz}), 123.4(\mathrm{q}, J=273.7 \mathrm{~Hz}), 38.1(\mathrm{q}, J=2.0 \mathrm{~Hz}) ;{ }^{19} \mathrm{~F}$ NMR $\left(376 \mathrm{MHz}, \mathrm{CDCl}_{3}\right) \delta:-41.4(\mathrm{~s}, 3 \mathrm{~F}),-62.9(\mathrm{~s}$, $3 \mathrm{~F})$.

辅助材料(Supporting Information) 详细的条件优化 表格，机理验证的实验步骤，以及化合物的表征谱图 (氢谱、碳谱、氟谱). 这些材料可以免费从本刊网站 (http:// sioc-journal.cn/)上下载.

\section{References}

[1] (a) Kirsch, P. Modern Fluoroorganic Chemistry, Wiley-VCH, Weinheim, 2004

(b) O'Hagan, D.; Rzepa, H. S. Chem. Commun. 1997, 645

(c) Müller, K.; Faeh, C.; Diederich, F. Science 2007, 317, 1881.

(d) Hagmann, W. K. J. Med. Chem. 2008, 51, 4359.

(e) Purser, S.; Moore, P. R.; Swallow, S.; Gouverneur, V. Chem Soc. Rev. 2008, 37, 320 .

(f) Zhu, H.; Liu, G. Acta Chim. Sinica 2012, 70, 2404 (in Chinese). (朱海涛, 刘国生, 化学学报, 2012, 70, 2404.)

(g) Lü, H.; Zhang, C.; Huang, J.; Zhao, Q.; Ma, Z.; Xiao, J. Acta Chim. Sinica 2012, 70, 1125 (in Chinese).

(吕会朝，张成潘，黄晋，赵巧玲，马志，肖吉昌，化学学报， 2012, 70, 1125.)

(h) Ni, C.; Zhu, L.; Hu, J. Acta Chim. Sinica 2015, 73, 90 (in Chinese).

(倪传法, 朱林桂, 胡金波, 化学学报, 2015, 73, 90.)

[2] (a) Leo, A.; Hansch, C.; Elkins, D. Chem. Rev. 1971, 71, 525.

(b) Yagupol'skii, L. M.; Il'chenko, A. Y.; Kondratenko, N. V. Russ. Chem. Rev. 1974, 43, 32.

(c) Hansch, C.; Leo, A.; Taft, R. W. Chem. Rev. 1991, 91, 165

(d) Leroux, F.; Jeschke, P.; Schlosser, M. Chem. Rev. 2005, 105 827.

(e) Manteau, B.; Pazenok, S.; Vors, J. P.; Leroux, F. R. J. Fluorine Chem. 2010, 131, 140

[3] (a) Yarovenko, N. N.; Vasileva, A. S. J. Gen. Chem. USSR (Engl. Transl.) 1958, 28, 2537.

(b) Nodiff, E.; Lipschutz, A. S.; Craig, P. N.; Gordon, M. J. Org. Chem. 1960, 25, 60.

(c) Feiring, A. E. J. Org. Chem. 1979, 44, 2907.

[4] (a) Billard, T.; Blond, G.; Langlois, B. R. Tetrahedron Lett. 1997, 38,65 .

(b) Billard, T.; Blond, G.; Langlois, B. R. Tetrahedron Lett. 2001, $42,2473$.

(c) Kieltsch, I.; Eisenberger, P.; Togni, A. Angew. Chem., Int. Ed. 2007, 46, 754.

[5] (a) Chen, P.; Liu, G. Synthesis 2013, 45, 2919.

(b) Yang, X.; Wu, T.; Phipps, R. J.; Toste, F. D. Chem. Rev. 2015, $115,826$.

(c) He, W.-M.; Weng, Z.-Q. Prog. Chem. 2013, 25, 1071 (in Chinese).

(何伟明，翁志强，化学进展, 2013, 25, 1071.)

(d) Zhang, K.; Xu, X.; Qing, F. Chin. J. Org. Chem. 2015, 35, 556 (in Chinese)

(张柯, 徐修华, 卿凤悀, 有机化学, 2015, 35, 556.)

(e) Xu, C.; Chen, Q.; Shen, Q. Chin. J. Chem. 2016, 34, 495.

(f) Chen, C.; Fu, L.; Chen, P.; Liu, G. Chin. J. Chem. 2017, 35 
1781.

(g) Zhang, P.; Lu, L.; Shen, Q. Acta Chim. Sinica 2017, 75, 744 (in Chinese).

(张盼盼, 吕龙, 沈其龙, 化学学报, 2017, 75, 744.)

[6] (a) Chen, Q.-Y.; Duan, J.-X. J. Chem. Soc., Chem. Commun. 1993, 918.

(b) Zhang, C.-P.; Vicic, D. A. Chem. Asian J. 2012, 7, 1756.

(c) Chen, C.; Chu, L.-L.; Qing, F.-L. J. Am. Chem. Soc. 2012, 134, 12454.

(d) Weng, Z.; He, W.; Chen, C.; Lee, R.; Dan, D.; Lai, Z.; Kong, D.; Yuan, Y.; Huang, K.-W. Angew. Chem., Int. Ed. 2013, 52, 1548 .

[7] Teverovskiy, G.; Surry, D. S.; Buchwald, S. L. Angew. Chem., Int. Ed. 2011, 50, 7312 .

[8] Chen, C.; Xie, Y.; Chu, L.; Wang, R.-W.; Zhang, X.; Qing, F. L. Angew. Chem., Int. Ed. 2012, 51, 2492.

[9] Zhang, C. P.; Vicic, D. A. J. Am. Chem. Soc. 2012, 134, 183.

[10] (a) Shao, X.; Wang, X.-Q.; Yang, T.; Lu, L.; Shen, Q. Angew. Chem., Int. Ed. 2013, 52, 3457.

(b) Pluta, R.; Nikolaienko, P.; Rueping, M. Angew. Chem., Int. Ed. 2014, 53, 1650 .

[11] Yin, G.; Kalvet, I.; Englert, U.; Schoenebeck, F. J. Am. Chem. Soc. 2015, 137, 4164.

[12] Yin, F.; Wang, X.-S. Org. Lett. 2014, 16, 1128.
[13] Ma, L.; Cheng, X.-F.; Li, Y.; Wang, X.-S. Tetrahedron Lett. 2016, $57,2972$.

[14] (a) Zhang, C.-P.; Wang, Z.-L.; Chen, Q.-Y.; Zhang, C.-T.; Gu, Y.-C.; Xiao, J.-C. Chem. Commun. 2011, 47, 6632.

(b) Lu, Q.-Q.; Liu, C.; Huang, Z.-Y.; Ma, Y.-Y.; Zhang, J.; Lei, A.-W. Chem. Commun. 2014, 50, 14101.

(c) Wang, F.; Wang, D.-H.; Mu, X.; Chen, P.-H.; Liu, G. J. Am. Chem. Soc. 2014, 136, 10202.

[15] (a) Huang, Y.-J.; He, X.; Lin, X.-X.; Rong, M.-G.; Weng, Z.-Q. Org. Lett. 2014, 16, 3284.

(b) Huang, Y.-J.; He, X.; Li, H.-H.; Weng, Z.-Q. Eur. J. Org. Chem. 2014, 7324

(c) Li, J.; Xie, F.-F.; Wang, P.-Q.; Wu, Q.-Y.; Chen, W.-D.; Ren, J.-M.; Zeng, B.-B. Tetrahedron 2015, 71, 5520.

(d) Wang, Y.-G.; You, Y.; Weng, Z.-Q. Org. Chem. Front. 2015, 2, 574.

(e) Wu, C.-Y.; Huang, Y.-J.; Chen, Z.-R.; Weng, Z.-Q. Tetrahedron Lett. 2015, 56, 3838 .

[16] (a) Xu, C.; Ma, B.; Shen, Q. Angew. Chem., Int. Ed. 2014, 53, 9316.

(b) Alazet, S.; Zimmer, L.; Billard, T. Chem.-Eur. J. 2014, 20, 8589.

[17] Yadav, A. K.; Singh, K. N. Chem. Commun. 2018, 54, 1976.

[18] Arimori, S.; Takada, M.; Shibata, N. Org. Lett. 2015, 17, 1063.

(Lu, Y.) 